\title{
Fekete-Szegö problem for a class of analytic functions defined by Carlson-Shaffer operator
}

\author{
Saurabh Porwal and Kaushal Kumar
}

\begin{abstract}
In the present paper, authors study a Fekete-Szegö problem for a class of analytic functions defined by Carlson-Shaffer operator. Relevant connections of the results presented here with various known results are briefly indicated.
\end{abstract}

Mathematics Subject Classification (2010): 30C45.

Keywords: Analytic function, Fekete-Szegö problem, Carlson-Shaffer operator.

\section{Introduction}

Let $A$ denote the class of functions $f$ of the form

$$
f(z)=z+\sum_{k=2}^{\infty} a_{k} z^{k}
$$

which are analytic in the open unit disk $U=\{z: z \in C$ and $|z|<1\}$ and $S$ denote the subclass of $A$ that are univalent in $U$. Fekete and Szegö [10] proved a interesting result that the estimate

$$
\left|a_{3}-\lambda a_{2}^{2}\right| \leq 1+2 \exp \left(\frac{-2 \lambda}{1-\lambda}\right)
$$

holds for any normalized univalent function $f(z)$ of the form (1.1) in the open unit disk $U$ for $0 \leq \lambda \leq 1$. This inequality is sharp for each $\lambda$.

The coefficient functional

$$
\phi_{\lambda}(f)=a_{3}-\lambda a_{2}^{2}=\frac{1}{6}\left(f^{\prime \prime \prime}(0)-\frac{3 \lambda}{2}\left[f^{\prime \prime}(0)\right]^{2}\right),
$$

on normalized analytic functions $f$ in the unit disk represents various geometric quantities, for example, when $\lambda=1, \phi_{\lambda}(f)=a_{3}-a_{2}^{2}$, becomes $\frac{S_{f}(0)}{6}$, where $S_{f}$ denote the Schwarzian derivative $\left(f^{\prime \prime \prime} / f^{\prime}\right)^{\prime}-\left(f^{\prime \prime} / f^{\prime}\right)^{2} / 2$ of locally univalent functions $f$ in 
$U$. The problem of maximising the absolute value of the functional $\phi_{\lambda}(f)$ is called the Fekete-Szegö problem.

The Fekete-Szegö problem is one of the interesting problems in Geometric Function Theory. This attracts many researchers (see the work of [1]-[5], [7]-[9], [12], [13], [16], [17], [20] and [3]) to study the Fekete-Szegö problem for the various classes of analytic univalent functions. Very recently, Bansal [4] introduced the class $R_{\gamma}^{\tau}(\phi)$ of functions in $f \in S$ for which

$$
1+\frac{1}{\tau}\left(f^{\prime}(z)+\gamma z f^{\prime \prime}(z)-1\right) \prec \phi(z), z \in U
$$

where $0 \leq \gamma<1, \tau \in C \backslash\{0\}, \phi(z)$ is an analytic function with positive real part on $U$ with $\phi(0)=0, \phi^{\prime}(0)>0$ which maps the unit like disk $U$ onto a starlike region with respect to 1 which is symmetric with respect to the real axis and $\prec$ denotes the subordination between analytic functions and studied the Fekete-Szegö problem for this class.

Now, by using the Carlson-Shaffer operator we introduce a new subclass $R_{\gamma}^{\tau}(\phi, a, c)$ for functions $f \in A$ and $0 \leq \gamma<1, \tau \in C \backslash\{0\}, a, c \in C,\{c \neq 0,-1,-2, \ldots\}$ satisfying the condition

$$
1+\frac{1}{\tau}\left((L(a, c) f(z))^{\prime}+\gamma z(L(a, c) f(z))^{\prime \prime}-1\right) \prec \phi(z) \quad(z \in U)
$$

where $\phi(z)$ is defined the same as above and $L(a, c)$ denotes the Carlson-Shaffer operator introduced in [6] and defined in the following way:

$$
L(a, c) f(z)=f(z) * z h(a, c ; z),
$$

where

$$
h(a, c ; z)=1+\sum_{n=1}^{\infty} \frac{(a)_{n}}{(c)_{n}} z^{n}
$$

$L(a, c)$ maps $A$ into itself. $L(c, c)$ is the identity and if $a \neq 0,-1,-2 \ldots$, then $L(a, c)$ has a continuous inverse $L(c, a)$ and is an one-to-one mapping of $A$ onto itself. $L(a, c)$ provides a convenient representation of differentiation and integration. If $g(z)=z f^{\prime}(z)$, then $g=L(2,1) f$ and $f=L(1,2) g$. If we set

$$
\phi(z)=\frac{1+A z}{1+B z}, \quad(-1 \leq B<A \leq 1 ; z \in U),
$$

in (1.4), we obtain

$$
\begin{gathered}
R_{\gamma}^{\tau}\left(\frac{1+A z}{1+B z}, a, c\right)=R_{\gamma}^{\tau}(A, B, a, c) \\
=\left\{f \in A:\left|\frac{(L(a, c) f(z))^{\prime}+\gamma z(L(a, c) f(z))^{\prime \prime}-1}{\tau(A-B)-B\left((L(a, c) f(z))^{\prime}+\gamma z(L(a, c) f(z))^{\prime \prime}-1\right)}\right|<1\right\}
\end{gathered}
$$

which is again a new class.

By specializing parameters in the subclass $R_{\gamma}^{\tau}(A, B, a, c)$ we obtain the following known subclasses studied earlier by various authors.

1. $R_{\gamma}^{\tau}(A, B, a, a) \equiv R_{\gamma}^{\tau}(A, B)$ studied by Bansal [4].

2. $R_{\gamma}^{\tau}(1-2 \beta,-1, a, a) \equiv R_{\gamma}^{\tau}(\beta)$ for $0 \leq \beta<1$, studied by Swaminathan [21]. 
3. $R_{\gamma}^{\tau}(1-2 \beta,-1, a, a) \equiv R_{\gamma}^{\tau}(\beta)$ for $\tau=e^{i \eta} \cos \eta, 0 \leq \beta<1$, where $-\pi / 2<\eta<\pi / 2$ introduced by Ponnusamy and Rønning [19], (see also [18]).

4. $R_{1}^{\tau}(0,-1, a, a) \equiv R^{\tau}(\beta)$ for $\tau=e^{i \eta} \cos \eta$ was considered in [14].

To prove our main result, we shall require the following lemma.

Lemma 1.1. (see [11], [15]). If $p(z)=1+c_{1} z+c_{2} z^{2}+c_{3} z^{3}+\ldots \quad(z \in U)$ is a function with positive real part, then for any complex number $\mu$,

$$
\left|c_{2}-\mu c_{1}^{2}\right| \leq 2 \max \{1,|2 \mu-1|\}
$$

and the result is sharp for the functions given by

$$
p(z)=\frac{1+z^{2}}{1-z^{2}}, \quad p(z)=\frac{1+z}{1-z} \quad(z \in U) .
$$

\section{Main results}

Our main result is contained in the following theorem.

Theorem 2.1. Let $\phi(z)=1+B_{1} z+B_{2} z^{2}+B_{3} z^{3}+\ldots$, where $\phi(z) \in A$ with $\phi^{\prime}(0)>0$. If $f(z)$ given by (1.1) belongs to $R_{\gamma}^{\tau}(\phi, a, c)(0 \leq \gamma \leq 1, \tau \in C \backslash\{0\}, a, c \in C$, $\{c \neq 0,-1,-2, \ldots\}, z \in U)$, then for any complex number $\mu$

$$
\left|a_{3}-\mu a_{2}^{2}\right| \leq \frac{B_{1}|\tau| c(c+1)}{3 a(a+1)(1+2 \gamma)} \max \left\{1,\left|\frac{B_{2}}{B_{1}}-\frac{3 \mu \tau B_{1} c(a+1)(1+2 \gamma)}{4 a(c+1)(1+\gamma)^{2}}\right|\right\} \text {. }
$$

This result is sharp.

Proof. If $f(z) \in R_{\gamma}^{\tau}(\phi, a, c)$, then there exists a Schwarz function $w(z)$ analytic in $U$ with $w(0)=0$ and $|w(z)|<1$ in $U$ such that

$$
1+\frac{1}{\tau}\left((L(a, c) f(z))^{\prime}+\gamma z(L(a, c) f(z))^{\prime \prime}-1\right)=\phi(w(z)), \quad(z \in U) .
$$

Define the function $p_{1}(z)$ by

$$
p_{1}(z)=\frac{1+w(z)}{1-w(z)}=1+c_{1} z+c_{2} z^{2}+\ldots \ldots
$$

Since $w(z)$ is a Schwarz function, we see that $\operatorname{Re}\left\{p_{1}(z)\right\}>0$ and $p_{1}(0)=1$.

Define the function $p(z)$ by,

$$
p(z)=1+\frac{1}{\tau}\left((L(a, c) f(z))^{\prime}+\gamma z(L(a, c) f(z))^{\prime \prime}-1\right)=1+b_{1} z+b_{2} z^{2}+\ldots
$$

In view of $(2.2),(2.3),(2.4)$, we have

$$
\begin{array}{r}
p(z)=\phi\left(\frac{p_{1}(z)-1}{p_{1}(z)+1}\right)=\phi\left(\frac{c_{1} z+c_{2} z^{2}+\ldots .}{2+c_{1} z+c_{2} z^{2}+\ldots . .}\right) \\
=\phi\left(\frac{1}{2} c_{1} z+\frac{1}{2}\left(c_{2}-\frac{c_{1}^{2}}{2}\right) z^{2}+\ldots . .\right) \\
=1+B_{1} \frac{1}{2} c_{1} z+B_{1} \frac{1}{2}\left(c_{2}-\frac{c_{1}^{2}}{2}\right) z^{2}+B_{2} \frac{1}{4} c_{1}^{2} z^{2}+\ldots .
\end{array}
$$


Thus,

$$
b_{1}=\frac{1}{2} B_{1} c_{1} ; \quad b_{2}=\frac{1}{2} B_{1}\left(c_{2}-\frac{c_{1}^{2}}{2}\right)+\frac{1}{4} B_{2} c_{1}^{2} .
$$

From (2.4), we obtain

$$
a_{2}=\frac{\tau B_{1} c_{1} c}{4 a(1+\gamma)} ; \quad a_{3}=\frac{\tau c(c+1)}{6 a(a+1)(1+2 \gamma)}\left[B_{1}\left(c_{2}-\frac{c_{1}^{2}}{2}\right)+\frac{1}{2} B_{2} c_{1}^{2}\right] .
$$

Therefore, we have

$$
a_{3}-\mu a_{2}^{2}=\frac{B_{1} \tau c(c+1)}{6 a(a+1)(1+2 \gamma)}\left(c_{2}-\nu c_{1}^{2}\right)
$$

where

$$
\nu=\frac{1}{2}\left(1-\frac{B_{2}}{B_{1}}+\frac{3 \tau \mu B_{1} c(a+1)(1+2 \gamma)}{4 a(c+1)(1+\gamma)^{2}}\right) .
$$

Our result now is followed by an application of Lemma 1.1. Also, by the application of Lemma 1.1 equality in (2.1) is obtained when

$$
p_{1}(z)=\frac{1+z^{2}}{1-z^{2}} \text { or } p_{1}(z)=\frac{1+z}{1-z}
$$

but

$$
p(z)=1+\frac{1}{\tau}\left((L(a, c) f(z))^{\prime}+\gamma z(L(a, c) f(z))^{\prime}-1\right)=\phi\left(\frac{p_{1}(z)-1}{p_{1}(z)+1}\right) .
$$

Putting value of $p_{1}(z)$ we get the desired results. Thus the proof of Theorem 2.1 is established.

For the class $R_{\gamma}^{\tau}(A, B, a, c)$,

$$
\phi(z)=\frac{1+A z}{1+B z}=(1+A z)(1+B z)^{-1}=1+(A-B) z-\left(A B-B^{2}\right) z^{2}+\ldots
$$

Thus, putting $B_{1}=A-B$ and $B_{2}=-B(A-B)$ in Theorem 2.1, we get the following corollary.

Corollary 2.2. If $f(z)$ given by (1.1) belongs to $R_{\gamma}^{\tau}(A, B, a, c)$, then

$$
\left|a_{3}-\mu a_{2}^{2}\right| \leq \frac{(A-B)|\tau| c(c+1)}{3 a(a+1)(1+2 \gamma)} \max \left\{1,\left|B+\frac{3 \tau \mu c(a+1)(A-B)(1+2 \gamma)}{4 a(c+1)(1+\gamma)^{2}}\right|\right\} \text {. }
$$

If we put $a=c$ in Theorem 2.1, then we obtain the following result of Bansal [4].

Corollary 2.3. Let $\phi(z)=1+B_{1} z+B_{2} z^{2}+B_{3} z^{3}+\ldots$, where $\phi(z) \in A$ with $\phi^{\prime}(0)>0$. If $f(z)$ given by (1.1) belongs to $R_{\gamma}^{\tau}(\phi)(0 \leq \gamma \leq 1, \tau \in C \backslash\{0\}, z \in U)$ then for any complex number $\mu$

$$
\left|a_{3}-\mu a_{2}^{2}\right| \leq \frac{B_{1}|\tau|}{3(1+2 \gamma)} \max \left\{1,\left|\frac{B_{2}}{B_{1}}-\frac{3 \mu \tau B_{1}(1+2 \gamma)}{4(1+\gamma)^{2}}\right|\right\} .
$$

This result is sharp. 


\section{References}

[1] Al-Abbadi, M.H., M. Darus, M., The Fekete-Szegö theorem for a certain class of analytic functions, Sains Malaysiana, 40(2011), no. 4, 385-389.

[2] Ali, R.M., Lee, S.K., Ravichandran, V., Supramaniam, S., The Fekete-Szegö coefficient functional for transform of analytic functions, Bull. Iran. Math. Soc., 35(2009), no. 2, 119-142.

[3] Al-Shaqsi, K., Darus, M., On the Fekete-Szegö problem for certain subclasses of analytic functions, Appl. Math. Sci., 2(2008), no. 8, 431-441.

[4] Bansal, D., Fekete-Szegö problem for a new class of analytic functions, Int. J. Math. Math. Sci., (2011), art. ID 143096, 1-5.

[5] Bhowmik, B., Ponnusamy, S., Wirths, K.J., On the Fekete-Szegö problem for concave univalent functions, J. Math. Anal. Appl., 373(2011), 432-438.

[6] Carlson, B.C., Shaffer, D.B., Starlike and Prestarlike hypergeometric functions, SIAM J. Math. Anal., 15(1984), 737-745.

[7] Cho, N.E., Owa, S., On Fekete-Szegö problem for strongly $\alpha$-quasiconvex functions, Tamkang J. Math., 34(2003), no. 1, 21-28.

[8] Choi, J.H., Kim, Y.C., Sugawa, T., A general approach to the Fekete-Szegö problem, J. Math. Soc. Japan, 59(2007), no. 3, 707727.

[9] Darus, M., Shanmugam, T.N., Sivasubramanian, S., Fekete-Szegö inequality for a certain class of analytic functions, Mathematica, 49(72)(2007), no. 1, 2934.

[10] Fekete, M., Szegö, G., Eine bemerkung uber ungerade schlichten funktionene, J. Lond. Math. Soc., 8(1993), 85-89.

[11] Keogh, F.R., Merkes, E.P., A coefficient inequality for certain classes of analytic functions, Proc. Amer. Math. Soc., 20(1969), 8-12.

[12] Koepf, W., On Fekete-Szegö problem for close-to-convex functions, Proc. Amer. Math. Soc., 101(1987), no. 1, 89-95.

[13] Koepf, W., On Fekete-Szegö problem for close-to-convex functions II, Archiv der Mathematik, 49(1987), no. 5, 420-433.

[14] Li, J.L., On some classes of analytic functions, Math. Japon., 40(1994), no. 3, 523-529.

[15] Libera, R.J., Zlotkiewicz, E.J., Coefficient bounds for the inverse of a function with derivative in $\rho$, Proc. Amer. Math. Soc., 87(1983), no. 2, 251-257.

[16] London, R.R., Fekete-Szegö inequalities for close-to-convex functions, Proc. Amer. Math. Soc., 117(1993), no. 4, 947-950.

[17] Murugusundaramoorthy, G., Kavitha, S., Rosy, T., On the Fekete-Szegö problem for some subclasses of analytic functions defined by convolution, Proc. Pakistan Acad. Sci., 44(2007), no. 4, 249-254.

[18] Ponnusamy, S., Neighbourhoods and Caratheodory functions, J. Anal., 4(1996), 41-51.

[19] Ponnusamy, S., Rønning, F., Integral transform of a class of analytic functions, Complex Var. Ellip. Equan., 53(2008), no. 5, 423-434.

[20] Shanmugan, T.N., Jeyaraman, M.P., Sivasubramanian, S., Fekete-Szegö functional for some subclasses of analytic functions, Southeast Asian Bull. Math., 32(2008), no. 2, 363370 .

[21] Swaminathan, A., Sufficient conditions for hypergeometric functions to be in a certain class of analytic functions, Computers Math. Appl., 59(2010), no. 4, 1578-1583. 
[22] Swaminathan, A., Certain sufficiency conditions on Gaussian hypergeometric functions, J. Inequal. Pure Appl. Math., 5(2004), no. 4, art. 83.

Saurabh Porwal

Department of Mathematics

UIET, CSJM University, Kanpur-208024

(U.P.), India

e-mail: saurabhjcb@rediffmail.com

Kaushal Kumar

Department of Mathematics

UIET, CSJM University, Kanpur-208024

(U.P.), India 